be strengthened by informed and uniform integration of STI testing within HIV programs, uptake of knowledge, awareness and health services much rely on engagement and involvement of vulnerable communities. Thus, enabling and building community response is critical in addressing issues pertaining to STI management. Community involvement can guide implementation and design for STI-focused interventions; strengthen on-ground outreach; identify barriers; and promote awareness of testing and treatment. As quality evidence is vital for informing strategies, it is critical to strengthen capacities and have communities' involvement in STI research. While community engagement and -stake may not address all challenges, it will be a solid step toward mitigating critical issues of low risk-perception and low uptake of STI testing and treatment among vulnerable communities.

\section{PL17 ENDING THE HIV EPIDEMIC: THE CRITICAL ROLE OF IMPLEMENTATION SCIENCE}

J Smith*. University of Utah School of Medicine, Salt Lake City, UT, USA

10.1136/sextrans-2021-sti. 17

In 2021, the requisite biomedical and behavioral interventions to eliminate new HIV infections exist. 'Ending the HIV Epidemic' worldwide now becomes primarily a challenge of will and implementation. For the past quarter century, the field of implementation science has been developing effective models and strategies to implement effective interventions in real-world service systems with the goal of efficiently providing interventions that reach the right people, at the right time, and in the right place. Yet, successful, sustained implementation is complex and requires a paradigmatic shift to focusing on the upstream factors in the system that are necessary to achieve the clinical effects demonstrated in efficacy research. While HIV/STI researchers have been implementing interventions for years, applying the best available scientific methods to this process has only recently begun to permeate the field and there are a number of gaps in knowledge, training, and application. This plenary will discuss the promising methods from implementation science for Ending the HIV Epidemic and describe some of the efforts in the United States to improve the science and lead to a world with no new HIV infections.

\section{PL18 MINORITY STRESS AND THE HEALTH OF LGBT POPULATIONS}

I Meyer*. UCLA School of Law, Los Angeles, USA

10.1136/sextrans-2021-sti.18

Minority stress theory describes how prejudice and stigma against LGBTQ people leads excess stress-both institutional and interpersonal-and, in turn, to adverse health outcomes. Social changes over the past 70 years in the United States have led to many changes in law and social attitudes toward LGBTQ people. In this presentation I will examine the impact of minority stress historically and today. I will report on results from two innovative U.S. national probability
samples-Generations and TransPop-of lesbian, bisexual, gay, and other sexual minorities (www.generationsstudy.com) and transgender adults (www.transpop.org). These studies cover topics related to identity and coming out, gender transition, exposure to chronic stress, life events, and everyday discrimination, and mental and physical health outcomes. We also examine current level of affiliation of LGBTQ people with the LGBTQ community-something that has been a key feature for delivering services and public health information to LGBTQ people at least since the 1980s. Results show that while we see many positive changes in society and, especially legal protections in the United States, these changes have not resulted in corresponding marked reduction in the experience of minority stress among LGBTQ people and have not reduced markers of poor health such as depression and suicidality. I will discuss the significance of these findings for public policy and future research in LGBTQ populations.

\section{Symposium presentations}

\section{S01.1 IDENTIFICATION OF BACTERIA ASSOCIATED WITH IDIOPATHIC NONGONOCOCCAL URETHRITIS}

S Srinivasan*. Fred Hutchinson Cancer Research Center, Seattle, USA

\subsection{6/sextrans-2021-sti.19}

Nongonococcal urethritis (NGU) is a common syndrome in men with no known etiology in up to $50 \%$ of cases. The most common causes include Chlamydia trachomatis (CT) and Mycoplasma genitalium (MG) accounting for 20\% to $40 \%$ and $10 \%$ to $30 \%$ of NGU cases respectively. Less common causes are Trichomonas vaginalis (TV), herpes simplex virus (HSV) and adenovirus. To identify if other bacteria are associated with NGU, we conducted a large study to estimate associations between urethral bacteria and NGU in men who have sex with men (MSM) and men who have sex with women (MSW). Urine samples were collected from 250 NGU cases and 184 controls attending a Seattle STD clinic. Cases had $\geq 5$ polymorphonuclear leukocytes on Gram stain plus symptoms or discharge; controls had $<5$ PMNs, no symptoms, no discharge. We characterized the urethral microbiota using $16 \mathrm{~S}$ rRNA gene sequencing, used compositional lasso analysis to identify associations between bacterial taxa and NGU and validated the associations with sensitive species-specific quantitative PCR. Among NGU cases, $45.2 \%$ were idiopathic, negative for known causes of NGU including CT, MG, TV, HSV and adenovirus. Our analysis revealed strong associations between detection of Haemophilus influenzae and idiopathic NGU among MSM $(20 \%$ vs. $3 \% ; \mathrm{p}=0.003)$ and MSW $(33 \%$ vs. $1 \% ; \mathrm{p}<0.001)$ while Mycoplasma penetrans was associated with NGU only among MSM $(25 \%$ vs. $1 \% ; \mathrm{p}<0.001)$ and not MSW $(5 \%$ vs. $1 \% ; \mathrm{p}=0.131)$. The two potential new causes of male urethritis, $H$. influenzae and $M$. penetrans, together accounted for an additional $18.8 \%$ of NGU cases in our study. Our current research is aimed at understanding the natural history and treatment outcomes for these bacteria in longitudinal samples that were collected daily and weekly in a subset of participants. Initial observations suggest that $\mathrm{M}$. penetrans can persist for several days post antibiotic therapy. 